PROCEEDINGS OF THE

AMERICAN MATHEMATICAL SOCIETY

Volume 126, Number 9, September 1998, Pages 2607-2614

S 0002-9939(98)04770-4

\title{
A UNIFIED EXTENSION OF TWO RESULTS OF KY FAN ON THE SUM OF MATRICES
}

\author{
TIN-YAU TAM
}

(Communicated by Lance W. Small)

\begin{abstract}
Let $A$ be an $n \times n$ Hermitian matrix with $\lambda(A)=\left(\lambda_{1}(A), \ldots\right.$, $\left.\lambda_{n}(A)\right)$ where $\lambda_{1}(A) \geq \cdots \geq \lambda_{n}(A)$ are the ordered eigenvalues of $A$. A result of Ky Fan (1949) asserts that if $A$ and $B$ are $n \times n$ Hermitian matrices, then $\lambda(A+B)$ is majorized by $\lambda(A)+\lambda(B)$. We extend the result in the framework of real semisimple Lie algebras in the following way. Let $\mathfrak{g}$ be a noncompact real semisimple Lie algebra with Cartan decomposition $\mathfrak{g}=\mathfrak{t}+\mathfrak{p}$. We show that for any given $p, q \in \mathfrak{p}, a_{+}(p+q) \leq a_{+}(p)+a_{+}(q)$, where $a_{+}(x)$ is the unique element corresponding to $x \in \mathfrak{p}$, in a fixed closed positive Weyl chamber $\mathfrak{a}_{+}$of a maximal abelian subalgebra $\mathfrak{a}$ of $\mathfrak{g}$ in $\mathfrak{p}$. Here the ordering $\leq$ is induced by the dual cone $\mathfrak{a}_{+}^{*}$ of $\mathfrak{a}_{+}$. Fan's result corresponds to the Lie algebra $\mathfrak{s l}(n, \mathbb{C})$. The compact case is also discussed. As applications, two unexpected singular values inequalities concerning the sum of two real matrices and the sum of two real skew symmetric matrices are obtained.
\end{abstract}

\section{INTRODUCTION}

A classical result of Fan (1949) asserts that if $A$ and $B$ are Hermitian matrices (denoted by $H_{n}$ ) with ordered eigenvalue element $\lambda(A)=\left(\lambda_{1}(A), \ldots, \lambda_{n}(A)\right.$ ) and $\lambda(B)=\left(\lambda_{1}(B), \ldots, \lambda_{n}(B)\right)$, where $\lambda_{1}(A) \geq \cdots \geq \lambda_{n}(A)$ and $\lambda_{1}(B) \geq \cdots \geq \lambda_{n}(B)$ are the eigenvalues of $A$ and $B$ respectively, then

$$
\lambda(A+B) \prec \lambda(A)+\lambda(B),
$$

i.e., majorization. The usual proof makes use of extremal representation (Marshall and Olkin, 1979, p.241). See Marshall and Olkin (1979) for an account of majorization and other inequalities associated with the sum of two Hermitian matrices.

Fan (1951) also proved that

$$
s(A+B) \prec_{w} s(A)+s(B),
$$

i.e., weak majorization, where $A$ and $B$ are $m \times n$ complex matrices (denoted by $\left.\mathbb{C}_{m \times n}\right)$ and $s(A)=\left(s_{1}(A), \ldots, s_{r}(A)\right), r=\min \{m, n\}$, denotes the singular value element of $A$, in which we have the ordering $s_{1}(A) \geq \cdots \geq s_{r}(A)$.

Received by the editors February 13, 1997.

1991 Mathematics Subject Classification. Primary 15A60, 22E30.

Key words and phrases. Eigenvalues, singular values, partial order.

Part of this work was done while the author was a visiting scholar in Mathematics Department of the University of Hong Kong, Dec. 1996-Jan. 1997. The travel was made possible by local subsistence provided by the department and travel grants from COSAM of Auburn University and NSF EPSCoR in Alabama. 
One way to get the weak majorization is through an observation of Wielandt (See Fan and Hoffman, 1955). If $A$ is an $m \times n$ complex matrix, then the nonzero eigenvalues of the Hermitian matrix

$$
\hat{A}=\left(\begin{array}{cc}
0 & A \\
A^{*} & 0
\end{array}\right)
$$

are the nonzero singular values of $A$ and their negatives. One may feel that the introduction of $\hat{A}$ is artifical.

The above two results of Fan may be cast in a form which makes sense for any real semi-simple Lie algebra $\mathfrak{g}$. Fan's first result corresponds to $\mathfrak{s l}(n, \mathbb{C})$ (more precisely, a translation is needed). The second result corresponds to $\mathfrak{s u}(m, n)$, in which Wielandt's $\hat{A}$ is quite a natural object. Moreover we will work out the results for a compact connected Lie group, in which $U(n)$ will give exactly Fan's result as we identify the Hermitian matrices with skew-Hermitian matrices.

We will consider two special examples, namely $\mathfrak{s o}_{n, n}$ and $\mathfrak{s o}(n)$, in which some new inequalities concerning the singular values of a sum are obtained.

\section{The NONCOMPACT CASE}

To describe our main results in this section we need to recall some basic notions from the theory of real semi-simple Lie algebra. Helgason (1978) is a good general reference. Let $\mathfrak{g}$ be a real semi-simple Lie algebra, i.e., a Lie algebra over the real field, with nondegenerate Killing form, and let $\mathfrak{g}=\mathfrak{k}+\mathfrak{p}$ be a Cartan decomposition. We assume that $\mathfrak{g}$ is noncompact, i.e., $\mathfrak{p} \neq 0$. The Killing form $\langle\cdot, \cdot\rangle$ is positive definite on $\mathfrak{p}$ and negative definite on $\mathfrak{k}$. Choose a maximal abelian subalgebra $\mathfrak{a}$ of $\mathfrak{p}$ and fix, once and for all, a closed Weyl chamber $\mathfrak{a}_{+}$in $\mathfrak{a}$. Let $K$ be the maximal compact subgroup of the adjoint group $\operatorname{Int}(\mathfrak{g})$. It is known that any element in $\mathfrak{p}$ is $\operatorname{Ad} K$ conjugate to an element in $\mathfrak{a}$, i.e., $k \cdot p=x$, for some $x \in \mathfrak{a}$ and some $k \in K$, where $p \in \mathfrak{p}$ and $k \cdot p$ denotes $\operatorname{Ad}(k) p$. Moreover, any $x \in \mathfrak{a}$ is $W$-conjugate to a unique element in $\mathfrak{a}_{+}$, where $W$ is the Weyl group of the pair $(\mathfrak{a}, \mathfrak{g})$ (the finite group defined as the quotient of the normalizer of $\mathfrak{a}$ in $K$ modulo the centralizer of $\mathfrak{g}$ in $K$; see Knapp (1986), p.126.) We also denote by conv $K p$ the convex hull of $K p=\{k \cdot p: k \in K\}$, the orbit of $p \in \mathfrak{p}$ for the $K$-action on $\mathfrak{p}$.

The dual cone of $\mathfrak{a}_{+}, \mathfrak{a}_{+}^{*} \subset \mathfrak{a}$, is defined by $x \in \mathfrak{a}_{+}^{*}$ if $\langle x, \gamma\rangle \geq 0$ for all $\gamma \in \mathfrak{a}_{+}$. The cone $\mathfrak{a}_{+}^{*}$ defines a natural partial order on $\mathfrak{a}$ by $x \geq y$ if $x-y \in \mathfrak{a}_{+}^{*}$. We will denote by $a_{+}(p)$ the unique element in the intersection of $K p$ and $\mathfrak{a}_{+}$.

There is a relation on $\mathfrak{a}$, defined by the Weyl group $W$ of the pair, such that $x \prec y$ if $x \in$ conv $W y$, the convex hull of the orbit of $y \in \mathfrak{a}$ under the action of $W$. The relation $\prec$ is equivalent to conv $W x \subset$ conv $W y$ and hence defines a partial order on the space of $W$-orbits of the elements in $\mathfrak{a}$.

Lemma 1. 1. (Kostant, 1973, Lemma 3.2) If $x \in \mathfrak{a}_{+}$, then $w \cdot x \leq x$ for any $w \in W$.

2. (Kostant, 1973, Lemma 3.3) If $x, y \in \mathfrak{a}_{+}$, then $x \leq y$ if and only if $x \prec y$.

3. If $x_{1}, x_{2}, y_{1}, y_{2} \in \mathfrak{a}$ and $x_{1} \leq y_{1}, x_{2} \leq y_{2}$, then $x_{1}+x_{2} \leq y_{1}+y_{2}$.

Theorem 2. If $x, y \in \mathfrak{p}$, then $a_{+}(x+y) \leq a_{+}(x)+a_{+}(y)$. 
Proof. Let $k \in K$ be such that $k \cdot(x+y)=a_{+}(x+y)$. Then for any $\gamma \in \mathfrak{a}_{+}$,

$$
\begin{aligned}
\left\langle a_{+}(x+y), \gamma\right\rangle & =\langle k \cdot(x+y), \gamma\rangle \\
& =\langle\pi(k \cdot(x+y)), \gamma\rangle \\
& =\langle\pi(k \cdot x), \gamma\rangle+\langle\pi(k \cdot y), \gamma\rangle,
\end{aligned}
$$

where $\pi: \mathfrak{p} \rightarrow \mathfrak{a}$ is the orthogonal projection with respect to the Killing form $\langle\cdot, \cdot\rangle$. By Kostant's convexity result (Kostant, 1973, Theorem 8.2), there exist nonnegative real numbers $\alpha_{i}, \beta_{i}$ and $w_{i} \in W, i=1, \ldots,|W|$, such that $\sum_{i=1}^{|W|} \alpha_{i}=\sum_{i=1}^{|W|} \beta_{i}=1$ and $\pi(k \cdot x)=\sum_{i=1}^{|W|} \alpha_{i} w_{i} \cdot a_{+}(x)$ and $\pi(k \cdot y)=\sum_{i=1}^{|W|} \beta_{i} w_{i} \cdot a_{+}(y)$. So by Lemma 1 ,

$$
\begin{aligned}
\langle\pi & (k \cdot x), \gamma\rangle+\langle\pi(k \cdot y), \gamma\rangle \\
& =\sum_{i=1}^{|W|} \alpha_{i}\left\langle w_{i} \cdot a_{+}(x), \gamma\right\rangle+\sum_{i=1}^{|W|} \beta_{i}\left\langle w_{i} \cdot a_{+}(y), \gamma\right\rangle \\
& \leq \sum_{i=1}^{|W|} \alpha_{i}\left\langle a_{+}(x), \gamma\right\rangle+\sum_{i=1}^{|W|} \beta_{i}\left\langle a_{+}(y), \gamma\right\rangle \\
& =\left\langle a_{+}(x), \gamma\right\rangle+\left\langle a_{+}(y), \gamma\right\rangle \\
& =\left\langle a_{+}(x)+a_{+}(y), \gamma\right\rangle .
\end{aligned}
$$

Hence $a_{+}(x+y) \leq a_{+}(x)+a_{+}(y)$.

We remark that $a_{+}(r x) \neq r a_{+}(x), x \in \mathfrak{a}, r \in \mathbb{R}$, e.g., $\lambda(-A) \neq-\lambda(A)$. For instance, $\lambda(-(2,1))=(-1,-2)$ but $-\lambda(2,1)=(-2,-1)$.

Example 3. Suppose that $\mathfrak{g}=\mathfrak{s}(n, \mathbb{C})$. We may take the Cartan decomposition

$$
\mathfrak{s l}(n, \mathbb{C})=\mathfrak{s u}(n)+\mathfrak{p},
$$

where $\mathfrak{p}$ is the space of traceless Hermitian matrices. The adjoint action of $K=$ $S U(n)$ on $\mathfrak{p}$ is given by $U \cdot A=U A U^{-1}$, where $U \in K=S U(n)$ and $A \in \mathfrak{p}$. A maximal abelian subalgebra in $\mathfrak{p}$ is

$$
\mathfrak{a}=\left\{\operatorname{diag}\left(\lambda_{1}, \ldots, \lambda_{n}\right): \sum_{i=1}^{n} \lambda_{i}=0\right\} .
$$

A closed Weyl chamber $\mathfrak{a}_{+}$in $\mathfrak{a}$ is given by

$$
\mathfrak{a}_{+}=\left\{\operatorname{diag}\left(\lambda_{1}, \ldots, \lambda_{n}\right): \sum_{i=1}^{n} \lambda_{i}=0, \lambda_{1} \geq \cdots \geq \lambda_{n}\right\} .
$$

The Killing form is $\langle A, B\rangle=2 n \operatorname{tr} A B$. So the restriction of the Killing form on $\mathfrak{a}$ is the usual inner product (up to a scalar multiple). Now

$$
\begin{aligned}
\langle x, y\rangle= & x_{1} y_{1}+\cdots+x_{n} y_{n} \\
= & \left(x_{1}-x_{2}\right) y_{1}+\left(x_{2}-x_{3}\right)\left(y_{1}+y_{2}\right) \\
& +\cdots+\left(x_{n-1}-x_{n}\right)\left(y_{1}+\cdots+y_{n-1}\right)+x_{n}\left(y_{1}+\cdots+y_{n}\right) .
\end{aligned}
$$

When $x_{1} \geq \cdots \geq x_{n}$, i.e., $x \in \mathfrak{a}_{+}$, the above computation shows that the cone $\mathfrak{a}_{+}^{*}$ is described by

$$
\sum_{i=1}^{k} y_{i} \geq 0, \quad k=1, \ldots, n-1, \quad \text { and } \quad \sum_{i=1}^{n} y_{i}=0 .
$$


In other words, $x \leq y$ means majorization while $x, y \in \mathfrak{a}_{+}$. Now if $x, y \in \mathfrak{a}$, then

$$
x \in \operatorname{conv} W y \Leftrightarrow a_{+}(x) \in \operatorname{conv} W a_{+}(y) \Leftrightarrow a_{+}(x) \leq a_{+}(y),
$$

by Lemma 1, i.e., majorization for $a_{+}(x)$ and $a_{+}(y)$. Of course, $a_{+}(x)$ amounts to the rearrangement of the entries of $x \in \mathfrak{a}$ in descending order. Thus Theorem 2 asserts that $\lambda(A+B) \prec \lambda(A)+\lambda(B)$, i.e., Fan's result after an appropriate translation, since $a_{+}(A)=\lambda(A)$.

Example 4. Suppose that $\mathfrak{g}=\mathfrak{s l}(n, \mathbb{R})$. We may take the Cartan decomposition

$$
\mathfrak{s l}(n, \mathbb{R})=\mathfrak{s o}(n)+\mathfrak{p},
$$

where $\mathfrak{p}$ is the space of traceless symmetric matrices. The adjoint action of $K=$ $S O(n)$ on $\mathfrak{p}$ is given by $U \cdot A=U A U^{-1}$, where $U \in K=S O(n)$ and $A \in \mathfrak{p}$. Now $\mathfrak{a}$ and $\mathfrak{a}_{+}$are chosen as in the previous example. The conclusion is similar to the previous example.

Example 5. Suppose that $\mathfrak{g}=\mathfrak{s u}_{m, n}, m \leq n$, which is the algebra (Onishchik and Vinberg, 1990)

$$
\begin{gathered}
\left\{\left(\begin{array}{cc}
X_{1} & Y \\
Y^{*} & X_{2}
\end{array}\right): \operatorname{tr} X_{1}+\operatorname{tr} X_{2}=0, X_{1}^{*}=-X_{1}, X_{2}^{*}=-X_{2},\right. \\
\left.X_{1} \in \mathbb{C}_{m \times m}, X_{2} \in \mathbb{C}_{n \times n}, Y \in \mathbb{C}_{m \times n}\right\} .
\end{gathered}
$$

We may take the Cartan decomposition

$$
\mathfrak{s u}_{m, n}=\mathfrak{k}+\mathfrak{p},
$$

where

$$
\begin{aligned}
\mathfrak{k} & =\left\{\left(\begin{array}{cc}
X_{1} & 0 \\
0 & X_{2}
\end{array}\right): X_{1}^{*}=X_{1}, X_{2}^{*}=X_{2}, \operatorname{tr} X_{1}+\operatorname{tr} X_{2}=0\right\}, \\
\mathfrak{p} & =\left\{\left(\begin{array}{cc}
0 & Y \\
Y^{*} & 0
\end{array}\right): Y \in \mathbb{C}_{m \times n}\right\} .
\end{aligned}
$$

Now

$$
K=\left\{\left(\begin{array}{cc}
U & 0 \\
0 & V
\end{array}\right): U \in U(m), V \in U(n), \operatorname{det} U \operatorname{det} V=1\right\} .
$$

The adjoint action of $K$ on $\mathfrak{p}$ is given by

$$
\begin{aligned}
\left(\begin{array}{cc}
U & 0 \\
0 & V^{*}
\end{array}\right) \cdot\left(\begin{array}{cc}
0 & A \\
A^{*} & 0
\end{array}\right) & =\left(\begin{array}{cc}
U & 0 \\
0 & V^{*}
\end{array}\right)\left(\begin{array}{cc}
0 & A \\
A^{*} & 0
\end{array}\right)\left(\begin{array}{cc}
U^{*} & 0 \\
0 & V
\end{array}\right) \\
& =\left(\begin{array}{cc}
0 & U A V \\
(U A V)^{*} & 0
\end{array}\right),
\end{aligned}
$$

where $\operatorname{det} U \operatorname{det} V^{*}=1, U \in U(m)$ and $V \in U(n)$.

A maximal abelian subalgebra $\mathfrak{a}$ in $\mathfrak{p}$ is

$$
\mathfrak{a}=\bigoplus_{i=1}^{m} \mathbb{R}\left(E_{i, m+i}+E_{m+i, i}\right)
$$

which can be identified with $\mathbb{R}^{m}$ in the obvious way, i.e., $E_{i, m+i}+E_{m+i, i} \mapsto e_{i}$, $i=1, \ldots, m$.

A closed Weyl chamber $\mathfrak{a}_{+}$of $\mathfrak{a}$ is given by

$$
\mathfrak{a}_{+}=\left\{\bigoplus_{i=1}^{m} a_{i}\left(E_{i, m+i}+E_{m+i, i}\right): a_{1} \geq \cdots \geq a_{m} \geq 0\right\},
$$


which can be identified with the cone $\left\{\left(a_{1}, \ldots, a_{m}\right): a_{1} \geq \cdots \geq a_{m} \geq 0\right\} \subset \mathbb{R}^{m}$. The Weyl group $G(m)$ acts on $\mathfrak{a}$ in such a way that

$$
\left(a_{1}, \ldots, a_{m}\right) \mapsto\left( \pm a_{\sigma(1)}, \ldots, \pm a_{\sigma(m)}\right),
$$

for any $\sigma \in \Sigma_{m}$ and any choice of sign, where $\left(a_{1}, \ldots, a_{m}\right) \in \mathbb{R}^{m}$ is viewed as an element of $\mathfrak{a}$. So if $x=\left(x_{1}, \ldots, x_{m}\right) \in \mathfrak{a}$, then $|x| \in \mathfrak{a}_{+}$, after we rearrange the entries of $x$ in descending order with respect to absolute value. The restriction of the Killing form of $\mathfrak{s u}_{m, n}$ on $\mathfrak{a}_{+}$is essentially the usual inner product. As before, we have

$$
\begin{aligned}
\langle x, y\rangle= & x_{1} y_{1}+\cdots+x_{m} y_{m} \\
= & \left(x_{1}-x_{2}\right) y_{1}+\left(x_{2}-x_{3}\right)\left(y_{1}+y_{2}\right) \\
& +\cdots+\left(x_{m-1}-x_{m}\right)\left(y_{1}+\cdots+y_{m-1}\right)+x_{m}\left(y_{1}+\cdots+y_{m}\right) .
\end{aligned}
$$

When $x_{1} \geq \cdots \geq x_{m} \geq 0$, i.e., $x \in \mathfrak{a}_{+}$, this shows that the cone $\mathfrak{a}_{+}^{*}$ is described by

$$
\sum_{i=1}^{k} y_{i} \geq 0, \quad k=1, \ldots, m .
$$

Thus $x \leq y$, where $x, y \in \mathfrak{a}_{+}$, means that $x \prec_{w} y$. Now if $x, y \in \mathfrak{a}$, then

$$
x \in \operatorname{conv} W y \Leftrightarrow a_{+}(x) \in \operatorname{conv} W a_{+}(y) \Leftrightarrow a_{+}(x) \leq a_{+}(y),
$$

i.e., $|x| \prec_{w}|y|$. So Theorem 2 implies that $s(A+B) \prec_{w} s(A)+s(B)$, since $a_{+}(A)=s(A)$.

\section{The COMPaCt CASE}

We now confine ourselves to compact connected Lie groups. Let $G$ be a compact connected Lie group with Lie algebra $\mathfrak{g}$. Let $T$ be a maximal torus of $G$ and let $\mathfrak{t}$ be the Lie algebra of $T$. Let $\mathfrak{t}_{+}$be a closed Weyl chamber in $\mathfrak{t}$. We fix once and for all a bi-invariant inner product $\langle\cdot, \cdot\rangle$ on $\mathfrak{g}$. We get a $W$-invariant inner product on $\mathfrak{t}$ and we define $\mathfrak{t}_{+}^{*}$ to be the dual cone of $\mathfrak{t}_{+}$, namely, $x \in \mathfrak{t}_{+}^{*}$ if $\langle x, y\rangle \geq 0$ for all $y \in \mathfrak{t}_{+}$. Following Atiyah and Bott (1982, p.600), we define the partial order on $\mathfrak{t}$ such that $x \geq y$ if $x-y \in \mathfrak{t}_{+}^{*}$. We also denote by $t_{+}(x)$ of the unique element in the intersection of the orbit $G x=\{g \cdot x: g \in G\}$ and $\mathfrak{t}_{+}$.

With these notions, all the previous results are valid if we replace $\mathfrak{p}$ by $\mathfrak{g}, K$ by $G, \mathfrak{a}$ by $\mathfrak{t}, \mathfrak{a}_{+}$by $\mathfrak{t}_{+}$, etc.

Lemma 6. 1. (Bourbaki 1968, Ch. VI, prop.18) If $x \in \mathfrak{t}_{+}$, then $w \cdot x \leq x$ for any $w \in W$.

2. (Atiyha-Bott 1982, Lemma 12.14) If $x, y \in \mathfrak{t}_{+}$, then $x \leq y$ if and only if $x \in \operatorname{conv} W y$.

3. If $x_{1}, x_{2}, y_{1}, y_{2} \in \mathfrak{t}$ and $x_{1} \leq y_{1}, x_{2} \leq y_{2}$, then $x_{1}+x_{2} \leq y_{1}+y_{2}$.

One can find Kostant's convexity theorem for a compact connected Lie group in Hilgert el at. (1989) or Atiyah and Bott (1982). So we have the following result.

Theorem 7. If $x, y \in \mathfrak{g}$, then $t_{+}(x+y) \leq t_{+}(x)+t_{+}(y)$.

Example 8. Let $G=U(n)$, the unitary group. The Lie algebra is $\mathfrak{u}(n)$, the set of skew Hermitian matrices, which can be identified with the set of Hermitian matrices via the map $A \in \mathfrak{u}(n) \mapsto i A \in H(n)$. Now we can pick $T=D(n) \subset U(n)$, the subgroup of diagonal matrices, as a maximal torus whose Lie algebra $\mathfrak{t}$ is the the set of diagonal skew Hermitian matrices. We then choose $\mathfrak{t}_{+}=\left\{i \operatorname{diag}\left(x_{1}, \ldots, x_{n}\right)\right.$ : 
$\left.x_{1} \geq \cdots \geq x_{n}\right\}$ as the closed Weyl chamber in $\mathfrak{t}$. Then $t_{+}(x+y) \leq t_{+}(x)+t_{+}(y)$ amounts to $\lambda(A+B) \prec \lambda(A)+\lambda(B)$, as we make the identification of $\mathfrak{u}(n)$ and $H(n)$, i.e., Fan's result.

\section{Applications: Singular values inequalities for a Sum of Real MATRICES OR OF REAL SKEW SYMMETRIC MATRICES}

Let $I_{n, n}=\left(-I_{n}\right) \oplus I_{n}$. The group $G=S O(n, n)$ is the group of matrices in $S L(2 n, \mathbb{R})$ which leaves invariant the quadratic form

$$
-x_{1}^{2}-\cdots-x_{n}^{2}+x_{n+1}^{2}+\cdots+x_{2 n}^{2} .
$$

In other words, $S O(n, n)=\left\{A \in S L(2 n, \mathbb{R}): A^{T} I_{n, n} A=I_{n, n}\right\}$. It is well known that

$$
\begin{aligned}
\mathfrak{s o}_{n, n} & =\left\{\left(\begin{array}{cc}
X_{1} & Y \\
Y^{T} & X_{2}
\end{array}\right): X_{1}^{T}=-X_{1}, X_{2}^{T}=-X_{2}, Y \in \mathbb{R}_{n \times n}\right\}, \\
K & =S O(n) \times S O(n), \\
\mathfrak{k} & =\mathfrak{s o}(n) \oplus \mathfrak{s o}(n), \text { i.e., } Y=0, \\
\mathfrak{p} & =\left\{\left(\begin{array}{cc}
0 & Y \\
Y^{T} & 0
\end{array}\right), Y \in \mathbb{R}_{n \times n}\right\}, \text { i.e., } X_{1}=X_{2}=0, \\
\mathfrak{a} & =\bigoplus_{1 \leq j \leq n} \mathbb{R}\left(E_{j, n+j}+E_{n+j, j}\right),
\end{aligned}
$$

where $E_{i, j}$ is the $2 n \times 2 n$ matrix where 1 at the $(i, j)$ position is the only nonzero entry. We will identify $\mathfrak{p}$ with $\mathbb{R}_{n \times n}$, the $n \times n$ real matrices. So $\mathfrak{a}$ and $\mathbb{R}^{n}$ are identified in the obvious way. We can take $\mathfrak{a}_{+}=\left\{\left(a_{1}, \ldots, a_{n}\right): a_{1} \geq \cdots \geq a_{n-1} \geq\right.$ $\left.\left|a_{n}\right|\right\} \subset \mathbb{R}^{n}$. Thus $a_{+}(A)=\left(s_{1}(A), \cdots, s_{n-1}(A)\right.$, sign $\left.\operatorname{det} A s_{n}(A)\right)$, where $s_{1}(A) \geq$ $\cdots \geq s_{n}(A)$ are the singular values of $A \in \mathbb{R}_{n \times n}$, which is viewed as $\mathfrak{p}$.

The Weyl group $W=S G(n)$, acting on $\mathfrak{a}$, can be viewed as $\Sigma_{n} \times(\mathbb{Z} / 2)^{n-1}$, and its action is given by the following:

$$
\left(\begin{array}{cc}
0 & D \\
D & 0
\end{array}\right) \in \mathfrak{a}, \quad\left(d_{1}, \ldots, d_{n}\right) \mapsto\left( \pm d_{\sigma(1)}, \ldots, \pm d_{\sigma(n)}\right),
$$

where $D=\operatorname{diag}\left(d_{1}, \ldots, d_{n}\right)$ and the number of minus signs is even.

Now Theorem 7 asserts that for any $A, B \in \mathbb{R}_{n \times n}$,

$$
\left(s_{1}(A+B), \ldots, s_{n-1}(A+B), \operatorname{sign} \operatorname{det}(A+B) s_{n}(A+B)\right)
$$

is in the convex hull of $S G(n)\left(a_{+}(A)+a_{+}(B)\right)$ where $a_{+}(A)+a_{+}(B)$ is

$$
\left(s_{1}(A)+s_{1}(B), \cdots, s_{n-1}(A)+s_{n-1}(B), \operatorname{sign} \operatorname{det} A s_{n}(A)+\operatorname{sign} \operatorname{det} B s_{n}(B)\right) .
$$

Then by a result in Tam (1997, Theorem 4$)$ which provides an equivalent statement in terms of inequalities, we have the following interesting inequalities concerning the singular values of a sum of $n \times n$ real matrices.

Theorem 9. Let $A, B \in \mathbb{R}_{n \times n}$ and $C=A+B$ with singular values arranged in descending order, e.g., $s_{1}(A) \geq \cdots \geq s_{n}(A) \geq 0$. Then

$$
\begin{gathered}
\sum_{i=1}^{k} s_{i}(C) \leq \sum_{i=1}^{k}\left(s_{i}(A)+s_{i}(B)\right), \quad k=1, \ldots, n-1, \\
\sum_{i=1}^{n} s_{i}(C) \leq \sum_{i=1}^{n-1}\left(s_{i}(A)+s_{i}(B)\right)+\left|\operatorname{sign} \operatorname{det} A s_{n}(A)+\operatorname{sign} \operatorname{det} B s_{n}(B)\right|,
\end{gathered}
$$




$$
\begin{aligned}
& \sum_{i=1}^{n-1} s_{i}(C)-s_{n}(C) \\
& \quad \leq \sum_{i=1}^{n-1}\left(s_{i}(A)+s_{i}(B)\right)-\left|\operatorname{sign} \operatorname{det} A s_{n}(A)+\operatorname{sign} \operatorname{det} B s_{n}(B)\right|
\end{aligned}
$$

and in addition, if ( $\left.\operatorname{sign} \operatorname{det} A s_{n}(A)+\operatorname{sign} \operatorname{det} B s_{n}(B)\right) \operatorname{det} C<0$, then

$$
\sum_{i=1}^{n} s_{i}(C) \leq \sum_{i=1}^{n-1}\left(s_{i}(A)+s_{i}(B)\right)-\left|\operatorname{sign} \operatorname{det} A s_{n}(A)+\operatorname{sign} \operatorname{det} B s_{n}(B)\right| .
$$

We notice that the inequalities (Fan 1951) $\sum_{i=1}^{k} s_{i}(C) \leq \sum_{i=1}^{k}\left(s_{i}(A)+s_{i}(B)\right)$, $k=1, \ldots, n$, are implied by the first two sets of inequalities of the result. Of course we can consider the Lie algebras $\mathfrak{s o}_{p, q}$ where $p \neq q$, but no new inequalities are obtained.

Another application is the compact connected Lie group $S O(2 n)$ whose Lie algebra is $\mathfrak{s o}(2 n)$, the algebra of real skew symmetric matrices. A maximal torus $T$ can be chosen as $S O(2) \oplus \cdots \oplus S O(2)$ whose Lie algebra $\mathfrak{t}$ is

$$
\left\{\left(\begin{array}{cc}
0 & \beta_{1} \\
-\beta_{1} & 0
\end{array}\right) \oplus \cdots \oplus\left(\begin{array}{cc}
0 & \beta_{n} \\
-\beta_{n} & 0
\end{array}\right): \beta_{1}, \ldots, \beta_{n} \in \mathbb{R}\right\},
$$

which can be identified with $\mathbb{R}^{n}$. Under this identification, we may choose a closed Weyl chamber $\mathfrak{t}_{+}=\left\{\left(\beta_{1}, \ldots, \beta_{n}\right): \beta_{1} \geq \cdots \geq \beta_{n-1} \geq\left|\beta_{n}\right| \geq 0\right\} \subset \mathbb{R}^{n}$.

For any $A \in \mathfrak{s o}(2 n)$, there exists $U \in S O(2 n)$ such that

$$
U A U^{-1}=\left(\begin{array}{cc}
0 & \beta_{1}(A) \\
-\beta_{1}(A) & 0
\end{array}\right) \oplus \cdots \oplus\left(\begin{array}{cc}
0 & \beta_{n}(A) \\
-\beta_{n}(A) & 0
\end{array}\right) \in \mathfrak{t}_{+},
$$

i.e., $\beta_{1}(A) \geq \cdots \geq \beta_{n-1}(A) \geq\left|\beta_{n}(A)\right|$. In other words, $t_{+}(A)=\beta(A) \equiv$ $\left(\beta_{1}(A), \ldots, \beta_{n}(A)\right)$. Clearly the numbers $\beta_{1}(A) \geq \beta_{1}(A) \geq \cdots \geq \beta_{n-1}(A) \geq$ $\beta_{n-1}(A) \geq\left|\beta_{n}(A)\right| \geq\left|\beta_{n}(A)\right|$ are the singular values of $A$. The Weyl group acts on $\mathfrak{t}$ exactly the same as in the case $\mathfrak{s o}_{n, n}$. Hence $\beta(A+B) \leq \beta(A)+\beta(B)$, or, equivalently, $\beta(A+B)$ is in the convex hull of $S G(n)(\beta(A)+\beta(B))$. So Theorem 7 gives the following result via Theorem 4 in Tam (1997).

Theorem 10. Let $A \in \mathfrak{s o}(2 n)$ and let $\beta(A)=\left(\beta_{1}(A), \ldots, \beta_{n}(A)\right)$, where

$$
U A U^{-1}=\left(\begin{array}{cc}
0 & \beta_{1}(A) \\
-\beta_{1}(A) & 0
\end{array}\right) \oplus \cdots \oplus\left(\begin{array}{cc}
0 & \beta_{n}(A) \\
-\beta_{n}(A) & 0
\end{array}\right)
$$

for some $U \in S O(2 n)$, with $\beta_{1}(A) \geq \cdots \geq \beta_{n-1}(A) \geq\left|\beta_{n}(A)\right|$. Let $A, B \in \mathfrak{s o}(2 n)$ and let $C=A+B$. Then

$$
\begin{aligned}
\sum_{i=1}^{k} \beta_{i}(C) & \leq \sum_{i=1}^{k}\left(\beta_{i}(A)+\beta_{i}(B)\right), \quad k=1, \ldots, n-1, \\
\sum_{i=1}^{n-1} \beta_{i}(C)+\left|\beta_{n}(C)\right| & \left.\leq \sum_{i=1}^{n-1}\left(\beta_{i}(A)+\beta_{i}(B)\right)+\mid \beta_{n}(A)+\beta_{n}(B)\right) \mid, \\
\sum_{i=1}^{n-1} \beta_{i}(C)-\left|\beta_{n}(C)\right| & \leq \sum_{i=1}^{n-1}\left(\beta_{i}(A)+\beta_{i}(B)\right)-\left|\beta_{n}(A)+\beta_{n}(B)\right|,
\end{aligned}
$$


and in addition, if $\beta_{n}(C)\left(\beta_{n}(A)+\beta_{n}(B)\right)<0$, then

$$
\sum_{i=1}^{n-1} \beta_{i}(C)+\left|\beta_{n}(C)\right| \leq \sum_{i=1}^{n-1}\left(\beta_{i}(A)+\beta_{i}(B)\right)-\left|\beta_{n}(A)+\beta_{n}(B)\right|
$$

The above inequalities depend on the orientation of the underlying real skew symmetric matrices $A, B$ and $C$. One may consider $\mathfrak{s o}(2 n+1)$, but only the weak majorization $s(A+B) \prec_{w} s(A)+s(B)$ can be deduced.

A larger class of inequalities (Thompson and Freede, 1971) for the sum of Hermitian matrices (complex matrices as well) are of the following form. Let $C=A+B$. Then

$$
\sum_{s=1}^{k} \lambda_{i_{s}+j_{s}-s}(C) \leq \sum_{s=1}^{k} \lambda_{i_{s}}(A)+\sum_{s=1}^{k} \lambda_{j_{s}}(B), \quad k=1, \ldots, n,
$$

where $1 \leq i_{1}<\cdots<i_{k} \leq n, 1 \leq j_{1}<\cdots<j_{k} \leq n, i_{k}+j_{k}-k \leq n$. It would be nice to extend those inequalities in the Lie framework.

\section{REFERENCES}

[A] Atiyah M. F. and Bott R. (1983), The Yang-Mills equations over Riemann surfaces, Phil. Trans. R. Soc. Lond. A 308:523-615. MR 85k:14006

[B] Bourbaki, N. (1968), Elements de mathematique, groupes et algébres de Lie, ch. 4-6. Paris, Hermann. MR 32:1590

[F1] Fan K. (1949), On a theorem of Weyl concerning eigenvalues of linear transformatioins I., Proc. Nat. Acad. Sci. U.S.A. 35:652-655. MR 11:600e

[F2] Fan K. (1951), Maximum properties and inequalities for the eigenvalues of completely continuous operators, Proc. Nat. Acad. Sci. U.S.A. 37:760-766. MR 13:661e

[F3] Fan K. and Hoffman A. (1955), Some metric inequalities in the space of matrices, Proc Amer. Math. Soc. 6:111-116. MR 16:784j

[He] Helgason S. (1978), Differential Geometry, Lie Groups and Symmetric Space, New York: Academic. MR 80k:53081

[Hi] Hilgert J., Hofmann K.H. and Lawson J.D. (1989), Lie Groups, Convex Cones and Semigroups, Oxford Science Publications. MR 91k:22020

[Ka] Knapp A. W. (1986), Representation Theory of Semisimple Groups, Princeton University Press, Princeton, New Jersey. MR 87j:22022

[Ko] Kostant B. (1973), On convexity, the Weyl group and Iwasawa decomposition, Ann. Sci. Ecole Norm. Sup. (4) 6:413-460. MR 51:806

[M] Marshall A. W. and Olkin I. (1979) Inequalities: Theory of Majorization and Its Applications, Academic Press, New York. MR 81b:00002

[O] Onishchik A.L. and Vinberg E. B. (1990), Lie Groups and Algebraic Groups, Springer-Verlag, Berlin. MR 91g:22001

[T] Tam T. Y. (1997), Kostant's convexity theorem and the compact classical groups, Linear and Multilinear Algebra 43 (1997), 87-113.

[Th] Thompson R. C. and Freede L. (1971), On the eigenvalues of sums of Hermitian matrices, Linear Algebra and Its Applications 4:369-376. MR 44:5330

Department of Mathematics, Auburn University, Auburn, Alabama 36849-5310

E-mail address: tamtiny@mail.auburn.edu 